\title{
Mobile Agent Based Approach Load Balancing in Heterogeneous Web Server System
}

\author{
Jyoti Vashistha ${ }^{1}$, Anant Kumar Jayswal ${ }^{2}$ \\ ${ }^{1}$ (Amity University,Noida) ${ }^{2}$ (Amity University, Noida)
}

\begin{abstract}
This Paper describes an approach of mobile agent based load balancing with highly heterogeneity and geographical distribution on web server system. Basically mobile agent integrated with various policies. The MPI is one of the primordial policies that is designed for model standard MPI library using java and compare with the mobile agent based approach load balancing. This also describes the performance evaluation of MALD and message passing based approach with different server execution.
\end{abstract}

Keywords: - PMADE, MALD Framework, Mobile Agent, Agent host, Agent Submitter.

\section{INTRODUCTION}

In heterogeneous web server system, the network load balancing provides scalability, high availability to enhance the performance of distributed web server system. The mobile agent based load balancing establish to efficient load balancing on wide range of heterogeneous web server system. Basically(on the whole) mobile agent are the software program that can move themselves from one network to another in heterogeneous network and Agent are intelligent software program that can be trusted(dependence) by their user to perform specific task on a certain type of data. In this paper, Mobile agent based approach have the capability to reduce the network traffic, provide greater flexibility, overcome network latency and also reduce network load. Mobile agent executes asynchronously and autonomously that adapt dynamically run independently in heterogeneous web server system.

To enhance the performance of distributed web server system the load balancing is an imperative technique that performs the various policies. Incoming client request should be evenly distributed among server to achieve quick response [1, 2].To enhance the system throughput the load on, overloaded server should be transferred to an under-loaded server. Therefore, the system resources are fully exploitated. Traditional load balancing approaches on distributed web servers are implemented based on message passing paradigm [1, 3].There are seven benefit to start a mobile agent for load balancing:-

1. Reduce the network load-

2. Overcome network latency

3. Encapsulate protocol

4. Executed asynchronous and autonomously

5. Adapt dynamically

6. Naturally heterogeneous

7. Robust and fault tolerant

\section{MALD FRAMEWORK}

The MALD framework is depend on a network of web servers that serves from LAN to WAN. Basically the servers define the hardware configuration, operating system and processing power with heterogeneous terms. The ability of server may change runtime due to the variation of workload. Every server can process client request individually and meanwhile cooperate with each other to share the workload.

Mobile Agent is a technology of combination of computer software and data which is proficiency migrate from one network to another asynchronously and autonomously and provide continue execution on destination computer. Mobile Agents execute only on the system where it starts execution and if need information on another system or need to interact with an agent on another system. They provide client server communication mechanism like as RPC, RMI, DCOM, CORBA 
Agent Specification

\begin{tabular}{|c|c|c|}
\hline SMA & \multicolumn{1}{|c|}{ Policy Specification } \\
\hline LIA & Information Gathering Policy \\
\hline JdA & Initiation Policy \\
\hline & Server Selection Policy \\
\hline & Job Transfer Policy \\
\hline
\end{tabular}

Fig 1 MALD FRAMEWORK

Mobile agent offer a technology for implementing load balancing mechanism on heterogeneous web server system. They can summarize the load balancing polices and travel to another node network that can make it decision on load distribution according to the latest state. The mobile agent load balancing specifies the three type of agents.

1. SMA(server management Agent)

2. LIA(load information Agent)

3. JDA(job delivering Agent )

Server Management Agent (SMA)-It is a stationary agent that motionlessly sits at a server, responsible for monitoring the workload on local server and executing job transfer policy if required. In sender initiated policy, when a server is overloaded, the SMA on it initiates the load reallocation process. The SMA selects the jobs from local job queue and dispatches the job to other servers.

Load Information Agent (LIA)- It is a mobile agent responsible for information gathering. It travels around servers, collects the load information and meanwhile propagates the load information to the servers. An LIA can be either a proprietary or a shared agent. A proprietary LIA belong to one server and works for it. LIA serves a load information for a group of servers.

Job Delivering Agent (JDA)-It is activated by the SMA on an overloaded server. A JDA executes the server selection policy to select another server to receive the reallocated job. Then the JDA carries reallocated job to that server and negotiates with it for the acceptance of the job. The JDA can also perform job redirection on the fly.

The implanted policies can deal with the load balancing requirements according to the up-to-date states of the servers and the client requests. The mobile agent based approach can minimize the network traffic and enhance the flexibility of load balancing mechanism. It define four policy-

1. Information Gathering policy-It define the method of information assembly policy and strategy for the collection of load information including the frequency. The frequency is determined based on the tradeoff between accuracy of load information and the overhead of information collection. Different scheme can be designed for this policy. Each server can dispatch its own mobile agent to collect load information. Or the server can share the information collected by common mobile agent.

2. Initiation Policy-It determines who will start the load balancing process. The process can be initiated by an overloaded server ( called sender initiative ) or by an under loaded server(called receiver initiative)

3. Server Selection Policy-Select an appropriate server based on the load information to which the workload on an overload server can be reallocated. Different strategies can be applied to the selection. For example, the find best strategy select the least load server among all servers and the find first strategy select the first server whose load is below a threshold.

4. Job Transfer policy-It determine when job reallocation should be performed and which job(i.e. client request)should be reallocated. Job reallocation is activated by threshold-based strategy. In a sender initiative method, the job transfer is invoked when the workload on server exceeds a threshold. In a receiver initiated method, a server start the process to fetch jobs from other servers when it workload is below to threshold. The threshold can be predefined static value or dynamic value that is assessed by runtime based on the load distribution among the servers. When reallocation is required, the appropriate job is selected from the job queue on a server and transferred to another server.

The mobile agent can interact with each other by direct data exchange. They can also interact the stigmergy in which the mobile agent can collect the information from the traces left in the environment by one another [4]. 


\section{PMADE ARCHITECTURE}

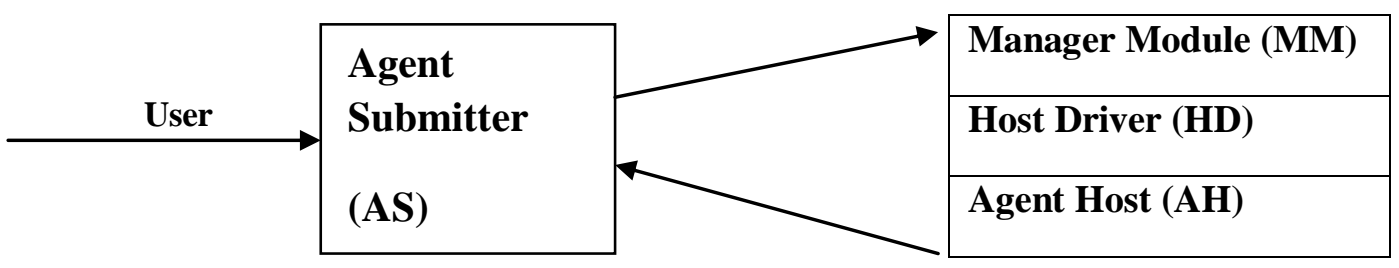

Fig 2 PMADE ARCHITECTURE

Fig 2. shows the basic block diagram of PMADE. The PMADE architecture consist of agent submitter(AS) i.e. responsible for accepting and executing incoming request of autonomous agent, java agent submitter(AS)[5]which is submit the mobile agent(user) to the AH. Basically AH is the key component of PMADE architecture, In which the user who want to complete the task, submit the mobile agent to perform that task to the agent submitter on the user system then ,the AS tries to establish the connection with respect to AH where the user already know about an account if the connection is establish then agent submitter submit the mobile agent to the user and then continue with that line execution. The agent host(AH) observe the behave of received agent then execute it. The agent execution depends on its environment and its state in which the transformation of agent can be performing from one state to another state whenever it required. When execution result have completed then the agent submit that result to the AH then store the result until the remote AS retrieve them for user. The agent submitter consists of manager module that is existed the above of PMADE and host driver that is lies base of the PMADE architecture. They are important module i.e. responsible for the execute the $\mathrm{AH}$ by ensuring proper interaction between them.

Details of the manager and their function are provided in [6]. PMADE provides weak mobility to its agent and allow one hop, two hop and multi hop agents [8].PMADE has focused on flexibility, persistence, security, collaboration and reliability [7].

\section{PERFORMANCE EVALUATION}

To study the MALD performance scheme on wide area network. We have developed a simulation environment using the IBM aglet 2.1.0 and JSDK(java 2) 1.4.1. The simulation of mobile agent allows the work of web server distributed on multiple agent by single PC. In which the load balancing perform the various policies i.e measured with following metrics

Network Traffic- The cluster represents the overall communication overhead i.e measured in the total no of bytes transferred in the communication.

Load Distribution-The Load Distribution of each server is considered by different time instants. Basically It defined by length of its job queue.

System Throughput-System throughput of web server, measured in the no. of request per second.

Fig 3 shows the system throughput of two approaches along $x$ axis number of client request is a factor of 100 while along y axis request satisfied/sec is shown. Clearly mobile agent approach is better than traditional message passing paradigm metric of the system throughput.

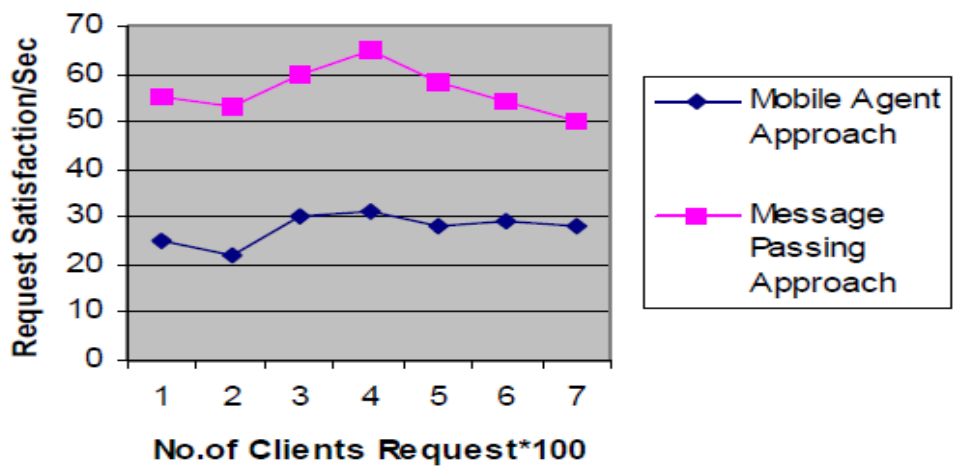

Fig 3 System Throughput

Fig 4 Compare the network traffic using mobile agent approach and message passing approach. It clearly shows that MA approach generates low communication delay compared to messaging passing approach. 


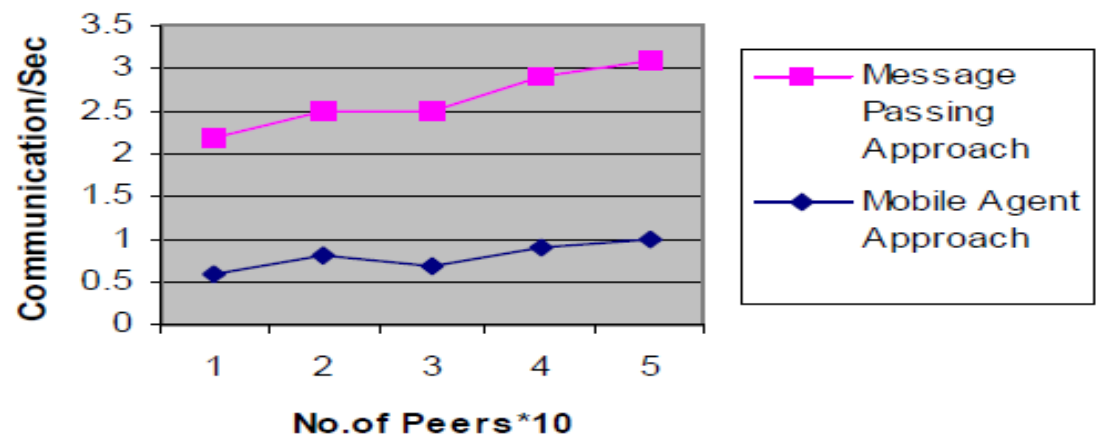

Fig 4 Network Traffic

\section{CONCLUSION AND FUTURE WORK}

In this study we have discussed different load balancing policies and MALD framework that based on mobile agent to support the load balancing on heterogeneous web server system and studied various these metrics affecting these policies using mobile agent approach. The performance evaluations of this approach shows that the mobile agent approach is better than the traditional based approach in the heterogeneous load balancing network. In the future work we would like to implement this approach to cluster of $\mathrm{PC}_{\mathrm{s}}$ and grid computing and also try to measure the different metrics regarding these systems for load balancing..

\section{REFERENCES}

[1] V. Cardellini, M. Colajanni, Dynamic Load Balancing on Web-server Systems, IEEE Internet Computing, 3 (1999), pp. 28-39.

[2] W. Tang, M. Mutka, Load Distribution via Static Scheduling and Client Redirection for Replicated Web Servers, in: Proc. 1st International Workshop on Scalable Web Services (in conjunction ICPP 2000), Toronto, Canada, 2000, pp. 127-133.

[3] D. Dias, W. Kish, R. Mukherjee, R. Tewari, A Scalable and Highly Available Web-Server, in: Proc. 41st International Computer Conference (COMPCON'96), IEEE Computer Society, San Jose, CA, 1996, pp. 85-92.

[4] N. Minar, K. Kramer, P. Maes, Cooperating Mobile Agents for Dynamic Network Routing, in: A.Hayzelden, (Ed.), Software Agents for Future Communication Systems, Springer-Verlag, 1999.

[5] Patel, R. B. and Garg, K., A New Paradigm for Mobile Agent Computing, WSEAS Transaction on Computers, Issue 1, Vol. 3, pp. 57-64, Jan. 2004.

[6] Patel, R.B. and Garg, K., PMADE - A Platform for mobile agent Distribution \& Execution, in Proceedings of 5th World MultiConference on Systemics, Cybernetics and Informatics (SCI2001) and $7^{\text {th }}$ International Conference on Information System Analysis and Synthesis (ISAS 2001), Orlando, Florida, USA, July 22-25, 2001, Vol. IV, pp. 287- 293.

[7] Patel, R. B., Design and Implementation of a Secure Mobile Agent Platform for Distributed Computing, $\mathrm{PhD}$ Thesis Department of Electronics and Computer Engineering, IIT Roorkee, India, Aug. 2004.

[8] Patel, R.B. and Garg, K, A Flexible Security Framework for Mobile Agent Systems Control and Intelligent Systems, 33(3): 175-183, 2005. 